\title{
Typical features of cerebellar ataxic gait
}

\author{
H Stolze, S Klebe, G Petersen, J Raethien, R Wenzelburger, K Witt, G DeuschI
}

J Neurol Neurosurg Psychiatry 2002;73:310-312

Background: Although gait disturbance is one of the most pronounced and disabling symptoms in cerebellar disease (CD), quantitative studies on this topic are rare.

Objectives: To characterise the typical clinical features of cerebellar gait and to analyse ataxia quantitatively.

Methods: Twelve patients with various cerebellar disorders were compared with 12 age matched controls. Gait was analysed on a motor driven treadmill using a three dimensional system. A tandem gait paradigm was used to quantify gait ataxia.

Results: For normal locomotion, a significantly reduced step frequency with a prolonged stance and double limb support duration was found in patients with $C D$. All gait measurements were highly variable in $C D$. Most importantly, balance related gait variables such as step width and foot rotation angles were increased in $C D$, indicating the need for stability during locomotion. The tandem gait paradigm showed typical features of cerebellar ataxia such as dysmetria, hypometria, hypermetria, and inappropriate timing of foot placement.

Conclusions: Typical features of gait in $C D$ are reduced cadence with increased balance related variables and an almost normal range of motion (with increased variability) in the joints of the lower extremity. The tandem gait paradigm accentuates all the features of gait ataxia and is the most sensitive clinical test.

A lthough cerebellar ataxic gait is a common and disabling symptom in many neurological diseases, systematic studies of the features of cerebellar gait are rare and the results contradictory. ${ }^{12}$ Clinical descriptions of cerebellar gait usually include a widened base, unsteadiness and irregularity of steps, and lateral veering. ${ }^{3}$ The patient may compensate for these abnormalities by shortening the steps and shuffling. ${ }^{3}$ In contrast with this clinical description, a quantitative study failed to show one of the key features, namely the widened base. ${ }^{1}$ The aim of our study was to analyse the typical features of cerebellar gait in order to quantify the variables that are most consistently disturbed. Therefore we focused especially on balance related features of gait during normal locomotion and after provoking ataxia in a routine clinical test (tandem gait paradigm).

\section{METHODS}

Twelve patients with cerebellar disease (CD) of various causes with a clear cut cerebellar syndrome were included in the study (mean age 47.9 years). All patients had gait disturbance of variable extent, limb ataxia, and intention tremor on neurological examination. Disease severity was mild to moderate, as no patient was wheelchair bound or used a rollator frame. Twelve age matched healthy subjects (mean age 47.3 years) served as controls. Patients and healthy subjects were excluded if either the history or the examination showed a hindrance or disease that interfered with an unrestrained gait, other than of cerebellar origin (for the patients). All subjects gave their informed consent to participate in the study, which was approved by the local ethics committee.

Gait analysis was carried out for two different tasks: (a) normal locomotion; $(b)$ tandem gait. The detailed method used to calculate the various gait variables, during both normal locomotion and tandem gait, has been described elsewhere. ${ }^{4}$ The natural walking speed, during normal locomotion and tandem gait, of each subject was measured during overground locomotion and was then preselected on the treadmill. During tandem gait, the subjects were instructed to place one foot in front of the other on a line of red tape that was attached to the lamella layers of the treadmill. Before recording, the subjects were given five minutes to familiarise themselves with treadmill locomotion. Gait was recorded with a three dimensional infrared movement analysis system (Qualisys, Sandvälen, Sweden). Seven infrared light reflective markers were attached to each leg. For each patient, 15-20 consecutive walking cycles were averaged for calculation of the different spatiotemporal gait measurements, including kinematics, with self developed software. ${ }^{5}$ During tandem gait, an ataxia ratio measuring the regularity of the strides in all three room directions was calculated as a ratio of the standard deviation (SD) of foot placement: (SD of step length $+\mathrm{SD}$ of step width $+\mathrm{SD}$ of step height) 3 . Missteps, defined as steps not hitting the tape, were counted over 60 seconds during tandem gait. For statistical analysis, the two groups were compared using the Mann-Whitney U test $(\mathrm{p}<0.05)$. Discriminant analysis was used to extract distinguishable gait variables in patients with $\mathrm{CD}$ and healthy controls.

\section{RESULTS}

\section{Normal walking}

Table 1 shows gait variables during normal and tandem gait. The most striking feature during normal locomotion was the large variability in $\mathrm{CD}$. The coefficients of variation of the step length (CD: 10.9 (SD 5.6)\%; controls: 5.5 (SD 2.0)\%), step height (CD: 9.8 (SD 3.7)\%; controls: 5.6 (SD 2.2)\%), and foot angles (CD: 39.0 (SD 17.2)\%; controls: 28.5 (SD 14.3)\%) were significantly higher for all variables in patients with CD. During normal walking, there was no difference in spontaneous gait velocity and stride length. Interestingly, the cadence was significantly lower in $\mathrm{CD}$, and the stance phase and double limb support duration showed that more time was spent in contact with the ground. Larger effects were found for the balance related gait variables, which are relevant for the maintenance of dynamic equilibrium during locomotion. Step width was significantly increased in $C D$, and the feet were rotated outward much more to improve stability. In most patients, either the step width or foot rotation angles were increased, which indicates individual strategies to improve balance. On the basis of these two variables, $87.5 \%$ of the subjects were correctly classified on discriminant analysis (Wilk's $\left.\lambda=0.4 ; \chi^{2}=17.5 ; \mathrm{p}<0.001\right)$. 
Table 1 Gait variables during normal locomotion and tandem gait in patients with cerebellar disease (CD) and healthy controls (HC)

\begin{tabular}{|c|c|c|c|c|}
\hline \multirow[b]{2}{*}{ Gait variables } & \multicolumn{2}{|l|}{ Normal gait } & \multicolumn{2}{|l|}{ Tandem gait } \\
\hline & $C D$ & $\mathrm{HC}$ & $C D$ & $\mathrm{HC}$ \\
\hline Velocity (m/s) & $0.96(0.31)$ & $1.11(0.18)$ & $0.29(0.8)$ & $0.27(0.5)$ \\
\hline Step length $(\mathrm{cm})$ & $53.7(16.1)$ & $56.3(7.2)$ & $27.9(5.5)$ & $25.3(1.9)$ \\
\hline Cadence (steps/min) & $106.4(14.2)^{*}$ & $119.1(14.4)$ & $67.2(10.8)$ & 69.7 (15.2) \\
\hline Step width $(\mathrm{mm})$ & $184(69)^{* *}$ & $116(43)$ & $48(45)^{\star * *}$ & $4(13)$ \\
\hline Step height $(\mathrm{mm})$ & $170(35)$ & 180 (17) & $106(24)$ & $14(50)$ \\
\hline Foot angle $\left({ }^{\circ}\right)$ & $17(4)^{*}$ & $13(3)$ & $11(7)$ & $9(4)$ \\
\hline Stance (ms) & $745(127) *$ & 638 (77) & $1403(233)$ & $1408(454)$ \\
\hline Swing (ms) & $403(51)$ & $383(51)$ & $440(70)$ & $468(88)$ \\
\hline DLS (ms) & $171(61) *$ & $128(23)$ & 605 (209) & $492(224)$ \\
\hline Hip ROM () & 26 (12) & $21(2)$ & & \\
\hline Knee ROM ( $\left.{ }^{\circ}\right)$ & $50(8)$ & $53(3)$ & & \\
\hline Ankle ROM (') & $25(5)$ & $19(4)$ & & \\
\hline Missteps/min & & & $8.2(11.5)^{* \star *}$ & $0.2(0.4)$ \\
\hline Ataxia ratio & & & $27.7(10.6)^{* * *}$ & $12.7(4.1)$ \\
\hline \multicolumn{5}{|c|}{$\begin{array}{l}\text { Values are mean }(S D)(n=12) \\
{ }^{*} p<0.05,{ }^{* *} p<0.01,{ }^{* * *} p<0.001 \text {, compared with healthy controls. } \\
\text { Stance, Stance phase duration; swing, swing phase duration; DLS, duration of the double limb support } \\
\text { phase; ROM, range of motion. }\end{array}$} \\
\hline
\end{tabular}
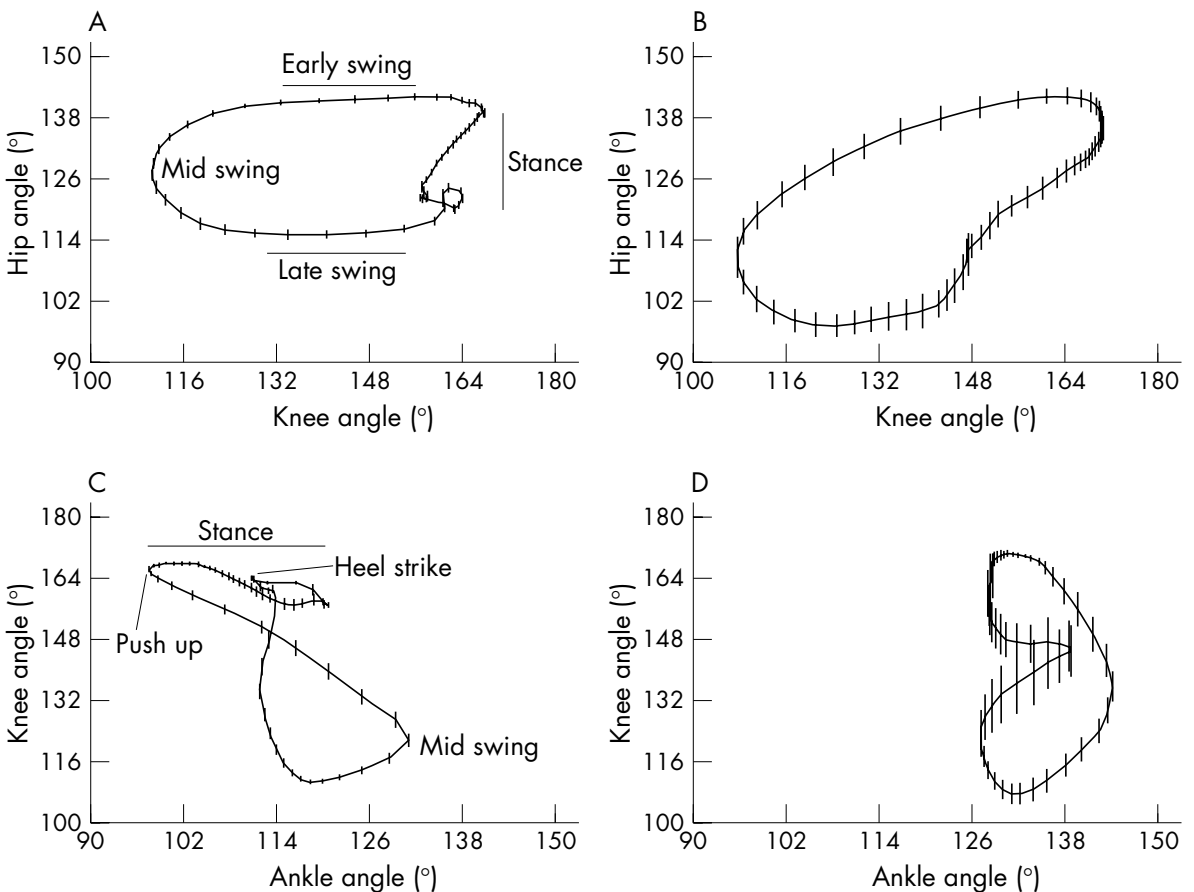

Figure 1 Angle-angle plots for hip-knee joint movements $(A, B)$ and knee-ankle joint movements $(C, D)$. $(A, C)$ Healthy control; $(B, D)$ patients with cerebellar disease (CD). Typical phases of the walking cycle are indicated for the healthy subject. Note the disturbed interlimb coordination in the patient with CD for the hip-knee angle plot, which shows that the hip and knee move pathologically in parallel throughout the gait cycle. In the knee-ankle angle plot for the patient with CD, it can be seen that ankle joint movements are restricted and there is insufficient dorsal extension of the forefoot.

The mean range of motion of all three joints of the lower extremity was not significantly different between patients with $\mathrm{CD}$ and controls. However, there was more variability in movements of the hip and knee joints in CD. Angle-angle plots (fig 1), which were very variable in $\mathrm{CD}$, also showed disturbed interlimb coordination of movement of the lower extremity joints.

\section{Tandem gait}

The critical point of tandem gait is the exact foot placement. Again most striking were the differences in the balance related gait variables ( table 1). The step width is given by the difference between the position of the two heels and should ideally be zero when the task is performed perfectly. In CD, the step width was often more than $5 \mathrm{~cm}$ and very variable. In patients with $C D$, the step width value was sometimes even negative, which means that one leg crosses the other during stance. Secondly, the ataxia ratio, which gives the variability in all the steps of an individual patient, is greatly increased in $\mathrm{CD}$, showing that the ideal path is often deviated from. Lastly, the number of missteps is significantly higher in CD. Gait ataxia is most strikingly visible if the pathway of markers attached to the lateral forefoot (both feet) is continuously recorded (fig 2). In healthy controls, the movement pattern is very regular, whereas in patients with CD it is irregular, with short and long steps, crossing of the legs, and deviation of the foot from the ideal pathway. 

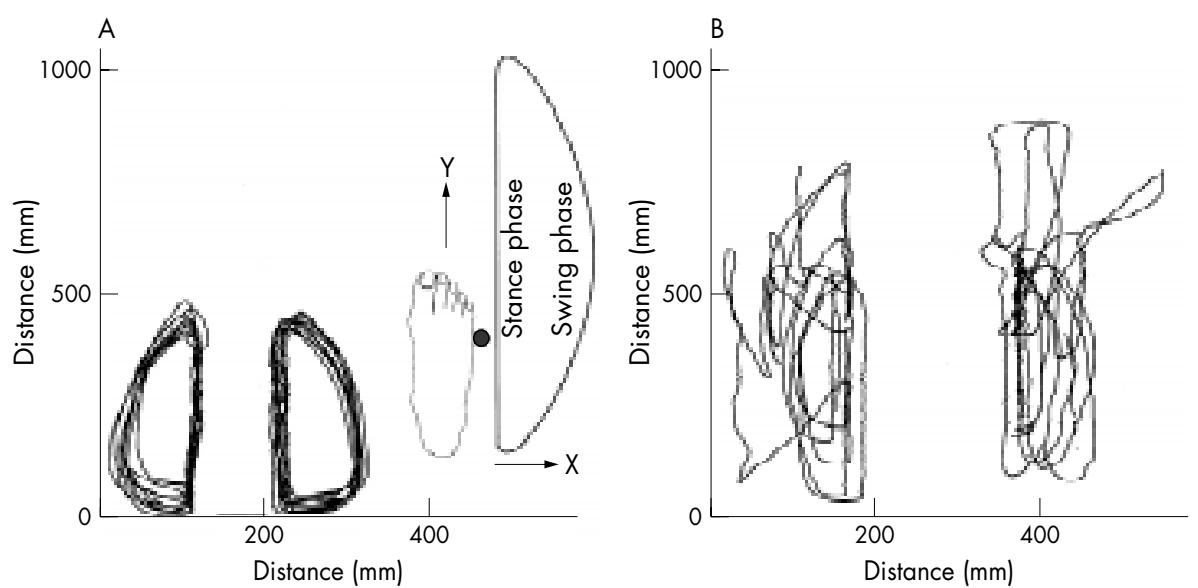

Figure 2 Direct tracings of markers attached in projection to the fifth metatarsal bone of both feet viewed from above (see schematic drawing in the middle) for a healthy subject (A) and a patient with cerebellar disease (B). Recordings were made during tandem walking on the treadmill over 20 seconds of continuous measurement. Note the dysmetric, hypometric, and hypermetric leg movements in the patient.

\section{DISCUSSION}

Movement analysis can give insights into sequences of movements that cannot usually be detected with the naked eye. Once the distinct key elements of abnormal movements are identified by movement analysis, this knowledge can be used to improve diagnostic skills in the daily clinical routine. Holmes ${ }^{6}$ has defined cerebellar ataxia as a combination of dysmetria, dyssynergia, dysdiadochokinesia, dysrhythmia, and intention tremor. The underlying phenomenon of dysmetria is force rate inadequacy and step amplitude misscaling. Dyssynergia is typical of multijoint movements, and has been studied for upper extremity movements ${ }^{78}$ and in gait. $^{19}$ Dyssynergia is described as decomposition of movements leading to abnormal movement path deviations, which are demonstrated typically in fig 2. Dysrhythmia describes the abnormal timing and coupling of movements, which we were able to show in our angle-angle plots (fig l).

Poor balance is a second major problem in $\mathrm{CD} .{ }^{10}$ Hitherto, balance related gait variables have been investigated in only one gait study, which failed to show a wide base. ${ }^{1}$ We here show poor balance in both normal walking and the tandem gait paradigm. In our sample of patients, gait velocity and stride length were almost normal. This is in contrast with the results of other gait studies of $\mathrm{CD},{ }^{12}$ probably because our patients had only mild to moderate gait impairment. Moreover, slowness of gait is a rather unspecific sign which all gait disorders have in common. We show that the cadence was reduced in $\mathrm{CD}$ as a result of increased stance and double limb support duration. This is clearly a compensatory mechanism to make walking more secure. ${ }^{1}$ In $\mathrm{CD}$ there is large variability in all gait parameters, probably because of imprecise movement execution. ${ }^{19}$ We show that balance related gait variables are altered in $\mathrm{CD}$ during normal walking and that step width and foot rotation angles are very different in patients with CD and healthy controls. This further supports the increased need for stability during walking in CD.

The most striking abnormalities in CD are found during tandem gait. The number of missteps is increased (more than one misstep per minute was never observed in healthy controls), the step width is large and far from the intended optimum, and the pathway of the foot during the task is very variable (ataxia ratio). Ataxia is best demonstrated by direct traces of the foot (fig 2), because dysmetria, hypometria, and hypermetria can be directly visualised. However, it is unlikely that this test can clearly differentiate between spinal and cerebellar ataxia and vestibular disease. Sometimes it is difficult to differentiate between a slight spastic paraplegia and ataxia. Here the tandem gait paradigm is helpful.

Taking all findings together, the gait disorder in CD is characterised by a widened base, increased foot rotation angles in combination with almost normal ranges of motion of the lower extremity, and relatively unchanged gait velocity and stride length, which decreases in severe or late stages of the disease. Tandem gait exaggerates dysmetria and balance problems and should be carried out in every patient with a gait disorder.

\section{Authors' affiliations}

H Stolze, S Klebe, G Petersen, J Raethien, R Wenzelburger, K Witt, $\mathbf{G}$ Deuschl, Department of Neurology, Christian-Albrechts-Universität Kiel, Niemannsweg 147, D-24105 Kiel, Germany

Correspondence to: Professor G Deuschl, Neurologische Klinik der Christian-Albrechts-Universität zu Kiel, Niemannsweg 147, 2405 Kiel, Germany; g.deuschl@neurologie.uni-kiel.de

Received 19 November 2001

In revised form 11 February 2002

Accepted 14 February 2002

\section{REFERENCES}

1 Palliyath S, Hallett M, Thomas SL, et al. Gait in patients with cerebellar ataxia. Mov Disord 1998;13:958-64.

2 Ebersbach G, Sojer M, Valldeoriola F, et al. Comparative analysis of gait in Parkinson's disease, cerebellar ataxia and subcortical arteriosclerotic encephalopathy. Brain 1999;122:1349-55.

3 Adams RD, Victor M, Ropper AH. Disorders of stance and gait. New York: McGraw-Hill, 1997.

4 Stolze $\mathbf{H}$, Petersen G, Raethien J, et al. The gait disorder of advanced essential tremor. Brain 2001;124:2278-86.

5 Stolze H, Kuhtz-Buschbeck JP, Mondwurf C, et al. Gait analysis during treadmill and overground locomotion in children and adults. Electroencephalogr Clin Neurophysiol 1997;105:490-7.

6 Holmes G. The cerebellum of man. Brain 1939;62:1-30

7 Thach WT, Perry JG, Kane SA, et al. Cerebellar nuclei: rapid alternating movement, motor somatotopy, and a mechanism for the control of muscle synergy. Rev Neurol 1993;149:607-28.

8 Topka H, Massaquoi SG, Benda N, et al. Motor skill learning in patients with cerebellar degeneration. J Neurol Sci 1998;158:164-72.

9 Earhart GM, Bastian AJ. Selection and coordination of human locomotor forms following cerebellar damage. J Neurophysiol 2001;85:759-69.

10 Diener HC, Dichgans J, Bacher M, et al. Quantification of postural sway in normals and patients with cerebellar diseases. Electroencephalogr Clin Neurophysiol 1984;57:134-42. 